\title{
Launderability of Surface-Insulated Cut and Sew E-Textiles
}

\author{
Md. Tahmidul Islam Molla, Crystal Compton, Lucy E. Dunne \\ University of Minnesota-Twin Cities, College of Design \\ Saint Paul, Minnesota, USA \\ \{islam056; ccompton; ldunne\}@umn.edu
}

\begin{abstract}
E-textiles that enable distribution of electronic components have advantages for wearable technology, in that functionality, power, and networking can be spread over a much larger area while preserving hand-feel and wearability. However, textile-embedded circuitry often must be machinewashable to conform to user expectations for care and maintenance, particularly for garments. In this study, we evaluate the robustness to home laundering of a previouslydeveloped cut-and-sew technique for assembling e-textile circuits. Alternative surface insulation materials, textile substrate properties, and soldered component joints are evaluated. After around 1000 minutes (16.67 hours) of rigorous washing and drying, we measured a best-case $0 \%$ failure rate for component solder joints, and a best-case 0.38 $\mathrm{ohm} / \mathrm{m}$ maximum increase in trace resistance. Liquid silicone seam sealer was effective in protecting $100 \%$ of solder joints. Two tape-type alternative surface insulation materials were effective in protecting bare traces and component attachment points respectively. Overall, results demonstrate the feasibility of producing insulated, washable cut-and-sew circuits for smart garment manufacturing.
\end{abstract}

\section{Author Keywords}

Encapsulation; embedded electronics; e-textiles; wearable technology; launderability; durability.

\section{ACM Classification Keywords}

J.2 Physical Sciences and Engineering (Electronics); J.6

Computer-Aided Engineering (Computer-Aided

Manufacturing).

\section{INTRODUCTION}

Embedded electronic technology has the potential to greatly enhance and augment the functionality of clothing and other wearable products. Many compelling applications like finegrained activity recognition, high-bandwidth and ergonomically placed wearable display, and haptic interfaces require that components be distributed over the body surface.

Permission to make digital or hard copies of all or part of this work for personal or classroom use is granted without fee provided that copies are not made or distributed for profit or commercial advantage and that copies bear this notice and the full citation on the first page. Copyrights for components of this work owned by others than ACM must be honored. Abstracting with credit is permitted. To copy otherwise, or republish, to post on servers or to redistribute to lists, requires prior specific permission and/or a fee. Request permissions from Permissions@acm.org.

ISWC '18, October 8-12, 2018, Singapore, Singapore

(C) 2018 Association for Computing Machinery.

ACM ISBN 978-1-4503-5967-2/18/10 .. \$15.00

https://doi.org/10.1145/3267242.3267255.
Textiles and clothing offer an advantage in networking, power, and usability for systems that rely on distributed functionality, but a key obstacle to garment-integration of technology is the tradeoff between durability and hand-feel or wearability. While consumers in general expect to be able to launder e-textile garments in the traditional manner, washability presents additional challenges for durability of e-textiles in two major ways [9]: first, the chemical interaction of the e-textile circuit with detergents and moisture, and second, the thermomechanical environment of the washer and dryer [16].

Even hydrophobic textile substrates can still transmit water through capillary effects and between interstices, which can make e-textiles vulnerable to water penetration [15]. While impermeable film laminates are used in some textile-based products, for many products impermeability is a severe wearability compromise (particularly in next-to-skin applications).

Textile-integrated rigid components are exposed to different levels of mechanical strain depending on the size of the components, textile architecture, and position of the components on the body [3]. For example, at the shoulder blades, tensile strain of up to $20 \%$ on integrated components has been observed in extensible (stretchy) garments [9]. Etextile components can be exposed to bending radii as small as millimeters (especially in strenuous environments like the tumble dryer), unlike flexible display applications which are exposed to bending radii of centimeters [3].

The traditional durability objective for e-textiles is to approximate the useful lifetime of traditional electronics [17]. Most existing approaches to improving the washability of e-textile garments require compromise of other interaction properties, particularly mechanical properties (but also properties like breathability and aesthetics). Further, effective integration with existing cut-and-sewn apparel production processes is important to the scalability of etextile manufacture. Here, we investigate the washability of an e-textile fabrication technique that aims to preserve textile and garment mechanical properties by minimizing the impact of integrated electronics. This is achieved through component distribution, stitched assembly methods, and minimization of integrated hard goods. We extend the technique to include approaches to encapsulation of integrated traces and components. Throughout, we emphasize methods that are easily deployable in typical cutand-sew manufacturing facilities. 


\section{BACKGROUND}

\section{Washability of E-Textiles}

A typical e-textile circuit consists of two major segments: a rigid area composed of electrical circuitry and a flexible area where conductive traces connect system components. Both of these segments need to be protected during regular wear and tear of a garment's life cycle. Most washability standards and studies pertain to the effects of washing on traditional textiles. There are few studies that have evaluated the washability of embedded electronics in textile applications.

Zeagler et al. [17] evaluated the effects of washing conductive materials commonly used in textile-based wearable applications, specifically focusing on electrical traces and capacitive touch sensitive devices fabricated entirely from textiles, yarns, and surface finishes. Based on average resistance change in the tested traces over 10 wash cycles, they found that using the plastisol ink improved durability by protecting the conductive ink traces. However, this study evaluated the durability and washability of embedded conductive traces and was not extended to affixed electronic components or supporting circuitry.

Schwarz et al. [12] evaluated the effects of washing on the resistance of elastic electro-conductive yarn (copper, silver, and stainless steel blended with elastomer). They found that conductivity of silver-based yarns remained constant after washing, however, washing decreased the conductivity of copper and stainless-steel yarns.

Li et al. [6] also investigated the robustness of textileintegrated conductive traces, this time polyurethane-coated copper fibers, in a home-laundering scenario. They found that electrical failures for samples washed without a protective bag began after 10 normal-intensity wash/dry cycles, with $15 \%$ of samples showing degradation after 30 cycles ( $11 \%$ for samples protected by a mesh bag). When washed using a delicate cycle, where no degradation was observed with or without a protective bag.

Dabby et. al. [4] used heat press to affix stretchable, waterproof bonding tape as an insulation to protect fabricbased electrodes in a biometric garment developed for heart monitoring. They performed total 25 wash tests using a traditional washing machine and the samples were washed using a medium-sized load in a regular was cycle with high and low agitation. Their results showed samples were fully functional up to 10 washing cycles and the performance started degrading significantly after that.

\section{E-Textile Protection Methods}

There are two popular approaches commonly used in the etextiles to protect sensitive electronic components from water and moisture. One approach is to make electronic components removable, so that they can be easily removed before washing [e.g. hexoskin.com]. Another approach is to apply a surface treatment barrier to insulate and/or encapsulate the electronic component and therefore protect from mechanical stresses and moisture. One of the major problems with surface treatment is that the extra protection layer can cause the garment surface to become stiff, impermeable, and/or otherwise uncomfortable to the user. Therefore, the materials used for encapsulation should be protective, lightweight, flexible, and comfortable. Further, compatibility with textile and garment manufacturing processes is important for scalability.

Many commonly-used in electronics fabrication techniques involve encapsulating components in a liquid-form impermeable coating. Epoxy resin or low-pressure injection hot melts can be used to seal small printed circuit boards (PCBs) [8]. A special UV curable resin can be used to seal a bare die on a PCB [8]. In larger areas, silicone and polyurethane (PU) encapsulations are more effective [5]. This approach is similar to the method developed by Linz et al. [7] for fabricating encapsulated, textile-integrated printed circuit boards where they used mold encapsulation (LOCTITE Hysol GR 9800) to protect a flexible PCB as well as the silver-coated polyamide traces.

Another approach to encapsulation is to apply an impermeable film on top of the protected area, on one or both sides. Berglund et al. [1] used fusible polymer film to insulate stretch sensor. PU films were used by the company Stretchable Circuits to encapsulate LEDs [14]. Welding spots can also be encapsulated using PU film [13].

Neijad et al. [11] developed a stretchable and washable thread-based piezo-resistive strain/force sensor to measure human physiological motion, which can be sewn into wearable textiles. To protect the sensor from environmental factors such as exposure to water and moisture, they used polymer coating (PDMS).

Tao et al. [15] used two different types of barrier protection methods to protect their electronic system. In their study, TPU films (thermoplastic polyurethane) were used to protect conductive thread and electrical contacts, in combination with a full-surface barrier made of latex. They found that TPU films did protect the electronic components, and stiffer silver-plated-silver copper tinsel thread showed better resistance performance compared to the more flexible silver polyamide thread and nickel-plated copper wire.

\section{METHODS}

In prior work, we developed a technique for reflow-type soldering of surface-mount electronic packages to stitched conductive traces [2]. In that study, the method was evaluated using two mechanical stress tests. We extended this work to refine the manufacturing technique and evaluate the durability of the resulting method [10]. In that study, the fabrication method had a component failure rate $3 \%$ after a 14-hour tumble dryer wear test.

In this study, the durability of the stitched fabrication method is extended to consider the effects of laundering, as well as the potentially mitigating effects of several circuit and protective barrier fabrication methods. Durability and wearability variables evaluated include: trace resistance, 
solder joint durability, encapsulation durability, and effect of e-textile and insulation components on textile stiffness.

\section{Textiles}

In the fabrication method used here, the mechanical and chemical properties of the substrate textile affect the performance and durability of the stitched e-textile circuit. For that reason, two different textiles were evaluated: $100 \%$ cotton and $100 \%$ polyester textile. Cotton (a natural, cellulosic fiber), is hydrophilic and more prone to shrinkage during washing and drying than polyester (a hydrophobic, synthetic fiber). Both textiles have the same structure (plain weave - twill variation) and are of similar weight (measured using the ASTM D3776 standard method).

Both textiles were pre-washed and dried once prior to circuit stitching and LED attachment, using the "Normal-Super Wash Casual/Permanent Press agitate/spin, Cold water" settings (60 min), and $60 \mathrm{~min}$ "Low" temperature dry cycle.

During the pre-wash process a shrinkage test was performed. The cotton fabric shrank $8.13 \%$ in the warp direction and $0 \%$ in the weft direction (this is because warp threads endure more tension during weaving and therefore are more prone to recovery-influenced shrinkage during washing and drying). The $100 \%$ polyester textile showed zero shrinkage after pre-washing and -drying.

\section{Electronic Component Conditions}

Two electronic component conditions were evaluated in this study to evaluate the influence of laundering on durability: surface-mount LEDs soldered to stitched traces, and stitched traces without an attached electronic component. Syscom Liberator ${ }^{\circledR} 40$ AG silver conductive thread (bobbin) with traditional $100 \%$ polyester all-purpose sewing thread (needle) was used for all traces. Five $3 \mathrm{~mm} 1206$ package LEDs were soldered onto the stitched traces of half of the samples for each fabric condition (Figure 1).

\section{Circuit Assembly Method}

Consistent with [10], a Brother BAS-342G Industrial Sewing Machine (Programmable Electronic Pattern Sewer) was used to stitch conductive traces in two-dimensional (2D) geometries, as shown in Figure 1.

For samples with traces and components, the two parallel stitched traces served as power and ground rails to which five $3 \mathrm{~mm}$ LEDs were attached, with the rail running perpendicular to the component package axis.

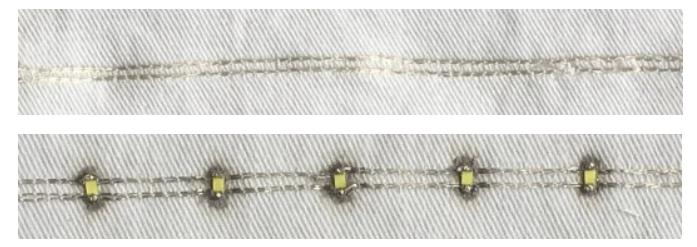

Figure 1. Stitched conductive traces using Programmable Electronic Pattern Sewer a) traces only, b) traces and soldered 3 mm LEDs.
Low-melt solder paste (Chip Quik $\AA$ ) mixed with an approximately equal amount of gel flux was screened onto the traces using a printmaking squeegee and a solder stencil, following the methods in [10]. LED packages were placed on the screened solder pads manually. To ensure even distribution of heat and pressure on the components, a PowerPress industrial plate heat press was used to reflow the solder paste. Once each swatch was fabricated, functionality of the LEDs was confirmed using a $5 \mathrm{~V}$ power source. $100 \%$ of swatches were functional following fabrication.

\section{Encapsulation of the Traces}

Twelve encapsulation materials (listed below) were initially evaluated in a pilot test. These materials emphasized two techniques: liquid encapsulation (consistent with traditional electronic encapsulation methods) and tape-type surface applications (consistent with traditional apparel seam reinforcements).

1. BEMISTM Seam Tape

2. Heat $n$ Bond $\AA$ Iron-on Adhesive, Ultrahold

3. Bondex $\AA / M D$ Mend \& Repair ${ }^{\mathrm{TM}}$ Fabric Mending Tape

4. Melco ${ }^{\mathrm{TM}}$ Iron-on Seam Tape 7/8"

5. Gear Aid Sil-Net ${ }^{\mathrm{TM}}$ Silicone Seam Sealer

6. Clover Quick Bias

7. SUNDLEY 20m Seam Sealing Tape

8. FRAMIS ITALIA S.P.A. black tape

9. Liquid electrical tape

10. Plasti Dip multi-purpose rubber coating

11. LED Seal Fast-Dry Spray (silicone spray sealant)

12. CRC Seal Coat clear urethane coating

Each material was applied to stitched conductive traces and washed and dried for one cycle. After preliminary testing of the initial 12 pilot samples, the five best performing materials (in terms of durability and stiffness) were selected to be used for further testing in this study, listed in Table 1. The selected materials prioritize those that can be applied in a fused taping operation (encapsulations 1-4), as taping machines are common in cut-and-sew fabrication facilities. The fifth material, liquid-type Sil-Net ${ }^{\mathrm{TM}}$ Silicone Seam Sealer, is included as a comparison method (as it is more similar to full-encapsulation methods used to protect electronics).

\begin{tabular}{|c|l|}
\hline ID & \multicolumn{1}{|c|}{ Name } \\
\hline (E1) & BEMIS $^{\mathrm{TM}}$ Seam Tape \\
\hline (E2) & $\begin{array}{l}\text { Layer 1: Heat } \mathrm{n} \text { Bond } \AA \text { Iron-on Adhesive, Ultrahold } \\
\text { Layer 2: Sheer synthetic textile }\end{array}$ \\
\hline (E3) & Bondex $^{\circledR} /$ MD Mend \& Repair ${ }^{\mathrm{TM}}$ Fabric Mending Tape \\
\hline (E4) & Melco $^{\mathrm{TM}}$ Iron-on Seam Tape 7/8" \\
\hline (E5) & Gear Aid Sil-Net $^{\mathrm{TM}}$ Silicone Seam Sealer \\
\hline
\end{tabular}

Table 1. Best-performing materials.

\section{Samples}

A total of 120 samples (containing a total of 300 LEDs, 600 solder joints and 240 traces) were fabricated for washability and durability testing. 
For each condition, a total of 20 samples were developed, five for each combination of textile/circuit variables (Cotton/trace only; Cotton/components; Poly/trace only; Poly/components). Five control samples were developed with no protective material (E0). Figures 2 and 3 illustrate the sample conditions evaluated in this study.

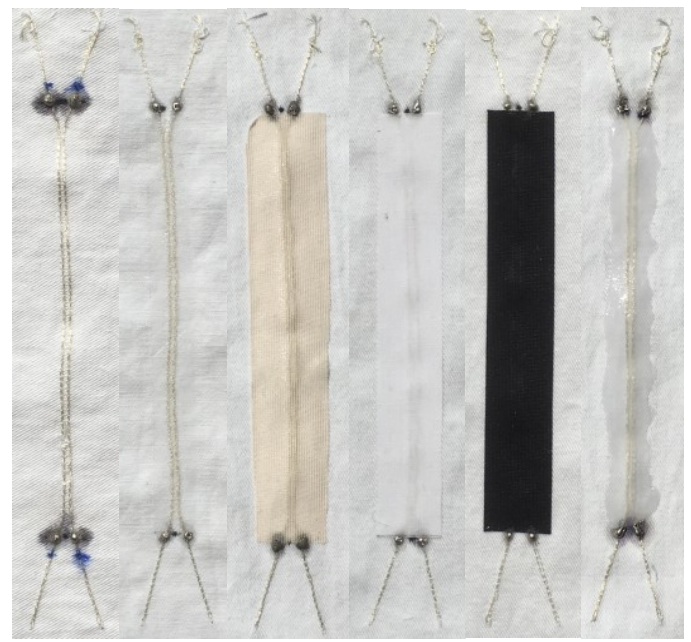

Figure 2. Trace conditions E0-E5.

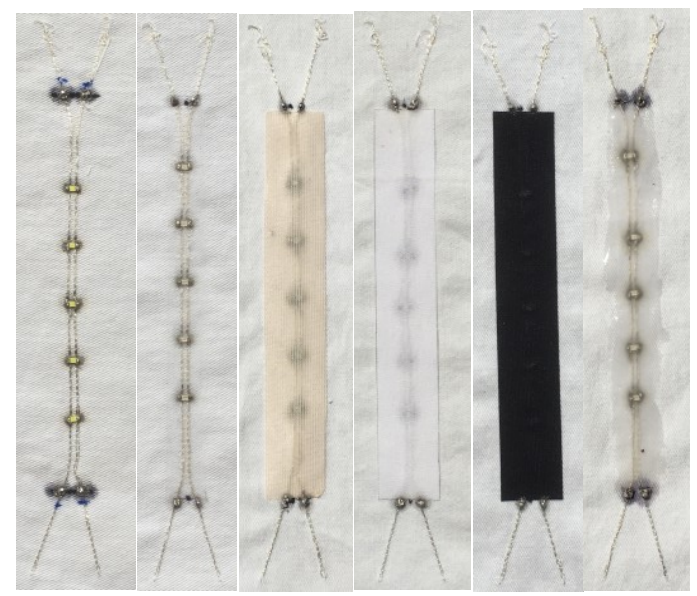

Figure 3. Trace and LED conditions E0-E5.

Each sample included a 2" border along the vertical (warp) and horizontal (weft) edges of the stitched circuit. The circuit measured approximately 7.25" in length (warp) and 1" (weft). The samples measured approximately 11.25 " in the warp direction and 5" in the weft direction. Raw edges were serged to prevent frayed edges from tangling during wash and dry cycles. Solder balls were applied to the ends of each trace outside the insulation region (5") to allow for standardized resistance testing.

\section{Barrier Materials: Method}

A PowerPress industrial plate heat press, also used for LED application, was used to fuse materials 1-4 (Figures 2 and 3) to the center of the stitched circuit on all textile samples. Each material measured 5" in length and varied in width dimensions $\left(0.5-0.75^{\prime \prime}\right)$.
Materials 1, 3, and 4 were heated to $400^{\circ} \mathrm{F}$ for 10 seconds during application. Material 2 was composed of a thermoplastic adhesive web (fused at $300^{\circ} \mathrm{F}$ for 10 seconds) used to adhere a textile layer (fused at $300^{\circ} \mathrm{F}$ for an additional 10 seconds).

Material 5 is applied as liquid silicone and does not require heat. This encapsulation was applied to the fabric using a small brush and cured for 6 hours.

To ensure that the encapsulation materials could withstand the temperature in the washing and drying machines, a pilot wash and dry test of all materials was performed using the washing and drying method described earlier. A total 10 samples were developed for each encapsulation material and samples were tested for one wash and dry cycle (total 100 minutes) to ensure they were able to withstand the machine temperature test settings.

\section{Fabric Stiffness Test Method}

The Peirce cantilever test method (ASTM D1388 standard) was used to determine the flexural rigidity of materials used in this study. This method measures the bend behavior of a material under its own weight at a $41.5^{\circ}$ angle.

One 8" (warp) by 1" (weft) sample was constructed for each non-LED condition, plus a textile sample with no stitched traces (14 samples total). The raw edges of these samples were not finished. Each sample was tested four times on the face and back side of the constructed sample ( 8 times total). The bent section of the sample was measured once it reached the $41.5^{\circ}$ angle of the testing device.

\section{Wash Testing Method}

The wash test method used was based on typical home laundering: machine washing and drying. Whirlpool ${ }^{\circledR}$ Ultimate Care II washing and tumble-drying machines were used for all wash and dry cycles in this study. All ${ }^{\circledR}$ "Free and Clear" detergent was used with all washing cycles.

For durability testing, all the samples underwent ten complete washing and drying cycles: 1000 minutes (approximately 16.67 hours) in total. To standardize the mechanical aspects of washing and drying for all samples we used the same water fill level (medium), temperature (cold setting, approximately 300C), detergent amount (2.4 oz.), and cycle time (40 minutes) during washing. For drying, same setting was used for all the samples e.g. temperature (low, approximately 300C) and cycle time (60 minutes). Washing at $300 \mathrm{C}$ is a common test for textiles and ISO approved [9] and cold temperature is typically used for soft and delicate fabric. After each wash and dry cycle, all swatches were removed and tested for LED functionality by applying the same $5 \mathrm{~V}$ power source. A digital multimeter (DMM) was used to measure the resistance of both traces using the four-wire resistance measurement method, which provides more reliable and consistent data and has been widely used in prior studies $[9,18,19]$. A total of 240 traces were measured for resistance after each washing and drying cycle. Trace resistance values and LED failures were 
recorded. Visible failed joints were photographed using a $30 \mathrm{X}$ microscope lens and categorized phenomenologically based on visual inspection.

\section{RESULTS}

\section{Stiffness Testing}

Averages and standard deviations were calculated for each sample condition tested across all stiffness test trials. Results are listed in Figure 4.

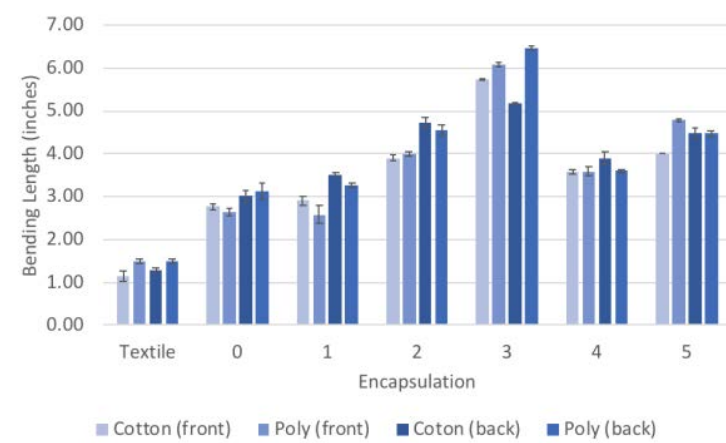

Figure 4. Average and standard deviation of bend length for each sample material: face- and back-side.

Of the two textile materials used in this study, the polyester fabric was stiffer compared to the cotton fabric. The addition of the protective layers to the fabric substrate increased its stiffness, as seen in Figure 4. For both textiles, material E3 was the stiffest. E3 was a fabric mending tape, in which the laminated fabric is stiffer fabric than our base substrates. (Although it was not measured, anecdotally this tape became more flexible after washing.) E1 was the most similar in flexibility to the un-insulated trace.

\section{Solder Joint Durability}

Figure 5 shows the number of LED failures observed for each condition after each wash cycle.
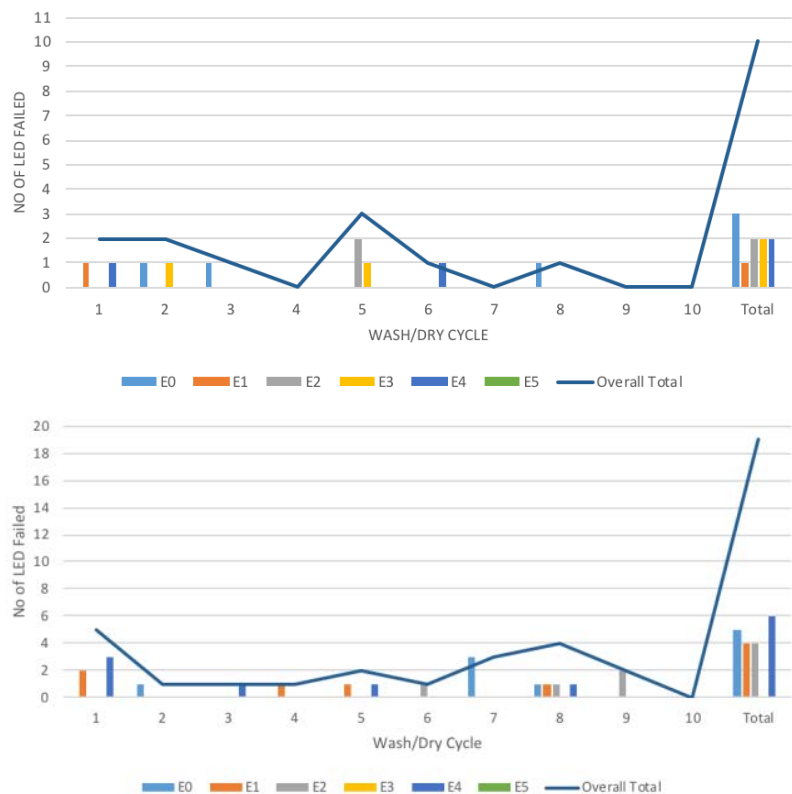

Figure 5. LED failures: cotton (top) and poly (bottom) samples
Trace Durability

Averages for resistance of all traces, in each encapsulation condition, for each wash cycle are shown in Figures 6-9.

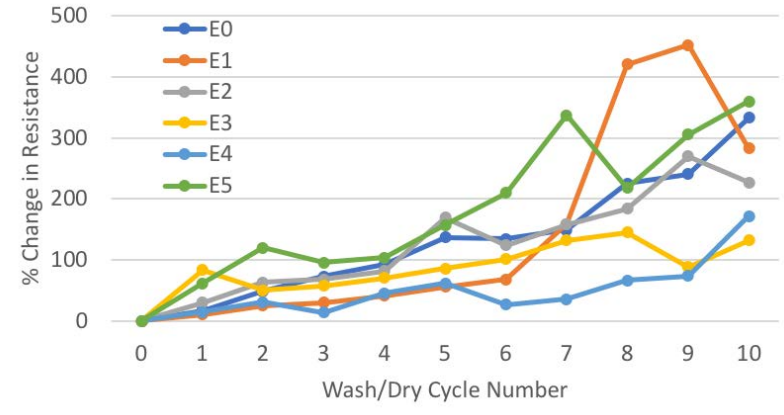

Figure 6. Average percent change in resistance for cotton

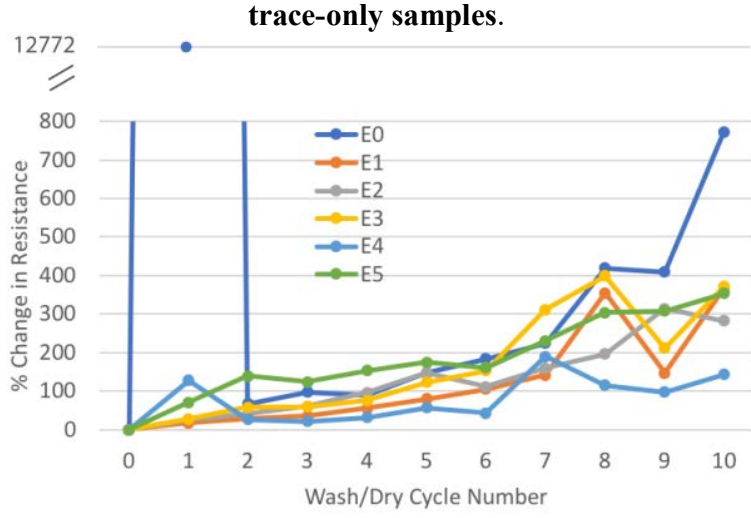

Figure 7. Average percent change in resistance for polyester trace-only samples.

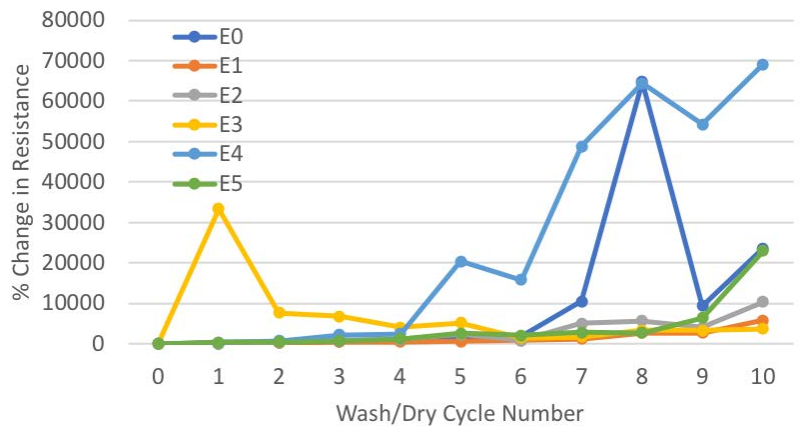

Figure 8. Average percent change in resistance for cotton LED samples.

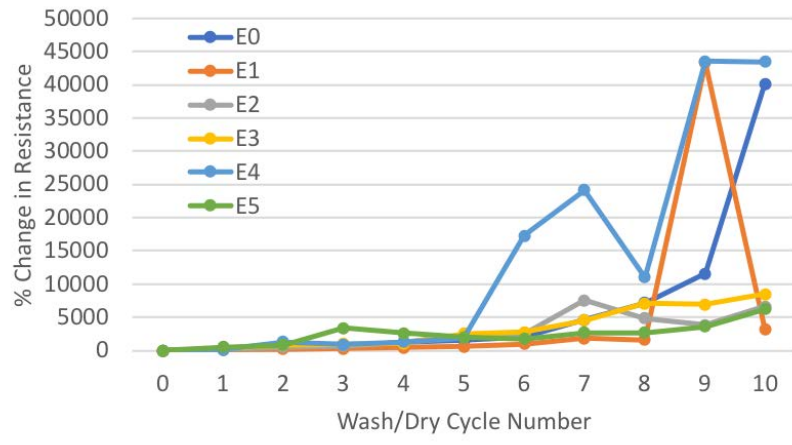

Figure 9. Average percent change in resistance for poly LED samples. 


\section{DISCUSSION}

Three trace durability metrics were measured: first, the instances of full breakage where irrecoverable damage resulted in a permanent open circuit. Second, temporary open circuits where large increases $(>8000 \Omega / \mathrm{m}$ approx.) in resistance were measured (intermittent effects are possible in multi-filament threads). Third, for each trace the maximum percentage increase in resistance over the testing period was calculated using the following formula:

$$
\left.\% \Delta R=\left(\left(R_{\max }-R_{i}\right) / R_{i}\right) * 100\right)
$$

This value was calculated using data from all trials where the trace resistance was measurable.

\section{Trace Resistance}

Overall, resistance of the traces increased as the number of cycles increased. However, trace resistance did not always increase steadily, it fluctuated between cycles.

\section{Effects of Fabric Substrate on Trace Resistance}

The average maximum change in resistance values of all the cotton samples (including both traces-only and traces with components) was lower than $(1144.73 \%, 1.79 \Omega / \mathrm{m}$ to 22.22 $\Omega / \mathrm{m})$ that of polyester fabric $(20172.39 \%, 1.71 \Omega / \mathrm{m}$ to $347.64 \Omega / \mathrm{m})$. The cotton textile conditions showed a lower resistance compared to the polyester textile conditions when no electronics were used. The cotton traces across all test cycles had lower average resistance values of $3.81 \Omega / \mathrm{m}$ (min: $1.67 \Omega / \mathrm{m}, \max : 10.06 \Omega / \mathrm{m}$, and SD: 1.88 ) across all the protective materials compared to the polyester traces which had an average resistance value of $8.13 \Omega / \mathrm{m}$ (min: 1.72 $\Omega / \mathrm{m}$, $\max : 238.39 \Omega / \mathrm{m}$, and SD: 28.89). This difference could potentially be due to the affinity for water between textiles. Cotton is a natural, hydrophilic fiber, thereby absorbing more moisture than a synthetic fiber, such as polyester, which has a lower affinity for moisture and is not able to absorb or diffuse water as much. However, as cotton fibers absorb water they swell, which may limit the transmission of water and detergent through the fabric-side of the sample, compared to hydrophobic polyester.

Interestingly, the cotton textile condition showed higher resistance compared to the polyester textile condition when components were attached. Cotton traces with LEDs across all test cycles had comparatively higher average resistance value of 140.46 (min:1.61 $\Omega / \mathrm{m}$, max: $1154.95 \Omega / \mathrm{m}$, and SD: 271.29) $\Omega / \mathrm{m}$ compared to the polyester traces with LEDs which had an average resistance value of $98.65 \Omega / \mathrm{m}$ (min: $1.58 \Omega / \mathrm{m}$, max: $799.31 \Omega / \mathrm{m}$, and SD: 187.76$)$. This could be an effect of the slightly higher stiffness of the polyester textile (see Fig 4), which may act as a slight strain-relief for solder joints.

Moreover, cotton fabric had more intermittent failures, but poly had more complete failures (breakages). There were 56 (out of 1200, 4.67\%) intermittent open circuit measurements in cotton samples along with 3 thread breakages. On the other hand, for the polyester fabric, there were $46(3.83 \%)$ intermittent open circuits and 7 instances of thread breakage. There was one intermittent open circuit measured for cottonstitched traces with LEDs and 6 for polyester-stitched traces with LEDs.

\section{Effects of Component Attachment on Trace Resistance}

More failures were noticed for traces with electronics compared to traces with no electronics. There were exactly $11(0.92 \%)$ intermittent open circuits for the trace-only samples compared to $91(7.58 \%)$ for the traces with components attached. However, the number of broken traces were same for both trace samples and LED samples (5). There were 7 intermittent open circuits, all observed in LED samples. For all conditions, trace-only samples had a lower overall resistance and lower variability (1144.73\% maximum increase of resistance over the initial resistance) than samples that contained traces with attached LEDs (avg: $119.55 \Omega / \mathrm{m}$, SD: 233.35). For un-protected trace-only samples, the maximum increase of resistance over initial value was $6573.16 \%(1.80 \Omega / \mathrm{m}$ to $120.22 \Omega / \mathrm{m})$, whereas for un-protected samples with LEDs the maximum increase was $32097.5 \%(1.65 \Omega / \mathrm{m}$ to $531.25 \Omega / \mathrm{m})$ which was much higher than the un-protected trace-only samples. The same pattern was observed for protected samples. For example, for BEMIS $^{\text {TM }}$ Seam Tape, the maximum change in the resistance value of the trace-only samples was much lower $(387.33 \%$, $1.82 \Omega / \mathrm{m}$ to $8.86 \Omega / \mathrm{m}$ ) than the samples with LEDs $(4416.13 \%, 1.76 \Omega / \mathrm{m}$ to $79.53 \Omega / \mathrm{m})$.

Cotton traces had lower maximum change in resistance $(251 \%, 1.74 \Omega / \mathrm{m}$ to $6.12 \Omega / \mathrm{m})$ than polyester traces $(2201.36 \%, 1.83 \Omega / \mathrm{m}$ to $42.07 \Omega / \mathrm{m})$ whereas cotton traces with LEDs had higher maximum resistance change $(22456.86 \%, 1.67 \Omega / \mathrm{m}$ to $375.68 \Omega / \mathrm{m})$ than polyester traces with LEDs $(19272.22 \%, 1.76 \Omega / \mathrm{m}$ to $341.76 \Omega / \mathrm{m})$. For the trace-only samples, Melco ${ }^{\mathrm{TM}}$ Seam Tape did the most to protect traces from breaking and/or increasing in resistance. On the other hand, Heat $n$ Bond ${ }^{\circledR}$ Iron-on Adhesive (E2), Sil-Net ${ }^{\mathrm{TM}}$ Silicone Seam Sealer (E5) and even, BEMIS ${ }^{\mathrm{TM}}$ Seam Tape (E1) (excluding one outlier) provided better performance in keeping the resistance values low for the traces with LEDs.

\section{Effects of Protection Method on Trace Resistance}

When no protective material was used, the increase of resistance was higher for traces with LEDs compared to traces with no LEDs. All five materials worked well to protect traces without LEDs compared to traces with LEDs. For all materials except Melco ${ }^{\mathrm{TM}}$ Seam Tape, the average resistances of the traces across all 120 samples (240 traces) were lower than the samples with no protective material. Heat $\mathrm{n}$ Bond $®$ Iron-on Adhesive provided the lowest average resistance value for all traces $(27.25 \Omega / \mathrm{m})$ followed by SilNet $^{\text {TM }}$ Silicone Seam Sealer $(28.26 \Omega / \mathrm{m})$, BEMIS tape $(30.70 \Omega / \mathrm{m})$, and Bondex ${ }^{\circledR}$ Fabric Mending Tape (45.4 $\Omega / \mathrm{m})$. The Melco ${ }^{\mathrm{TM}}$ Seam Tape samples had higher average resistance $(168.35 \Omega / \mathrm{m})$ than the unprotected samples $(76.98$ 
$\Omega / \mathrm{m})$. Overall, protected samples had lower average resistance $(59.92 \Omega / \mathrm{m})$ compared to unprotected samples. Protection helped more for the trace-only samples compared to LED samples (the changes in the maximum average resistance values were much lower for trace-only samples compared to LED samples.)

Influence of the various materials used here on trace and solder joint durability were mixed. The results showed that Bondex ${ }^{\circledR}$ Fabric Mending Tape (E3) and Melco ${ }^{\mathrm{TM}}$ Seam Tape (E4) performed well while protecting the cotton traces with no electronics from degrading whereas BEMIS tape (E1), Heat $\mathrm{n}$ Bond ${ }^{\circledR}$ Iron-on Adhesive (E2) and Sil-Net ${ }^{\mathrm{TM}}$ Silicone Seam Sealer (E5) performed well when electronics were attached. For polyester fabric, all materials helped to reduce the resistance change of the traces, but Melco ${ }^{\text {TM }}$ Seam Tape (E4) provided the best results when no electronics were used and Sil-Net ${ }^{\mathrm{TM}}$ Silicone Seam Sealer (E5), followed by Heat $n$ Bond ${ }^{\circledR}$ Iron-on Adhesive (E2), Melco ${ }^{\text {TM }}$ Seam Tape (E4) and even BEMIS tape (E1) (excluding one outlier) provided similarly strong performance for traces with soldered components. In summary, Melco ${ }^{\mathrm{TM}}$ Seam Tape (E4) provided the best results for protecting traces with no components. BEMIS tape (E1) and Sil-Net ${ }^{\mathrm{TM}}$ Silicone Seam Sealer (E5) provided the best results for protecting cottonLED traces and polyester-LED traces respectively. Interestingly, while Melco seam sealer provided the best results to limit the increase of the resistance of the traces with no electronics, it also provided the worst results for both cotton-LED and polyester-LED samples.

\section{Limitations}

While measuring the resistance values using the digital multimeter (DMM), we applied force using the DMM probes to provide a consistent measurement context. This repeated force may have contributed to thread breakages but was experienced in the same way by all samples. Due to the same repeated force, some of the small beads of solder we used on each trace end to get consistent resistance measurements were came off from the threads after few washing and drying cycles. Subsequent measurements were taken in the same locations without the benefit of the stable surface, which may have affected some measurements.

\section{Durability of Solder Joints}

Of all of the protective materials, Sil-Net ${ }^{\mathrm{TM}}$ Silicone Seam Sealer showed the best results with zero failure. Sil-Net ${ }^{\mathrm{TM}}$ Silicone Seam Sealer is very popular as an encapsulation material in the electronics world and is widely used to protect vulnerable electronic connections, but is less well-suited to the cut-and-sewn manufacturing environment. Of the tapetype materials, Bondex ${ }^{\circledR}$ Fabric Mending Tape was the best for protecting solder joints $(2 \%)$, followed by BEMISTM Seam Tape $(6 \%)$ and Heat $n$ Bond ${ }^{\circledR}$ Iron-on Adhesive $(6 \%)$. Melco $^{\mathrm{TM}}$ Seam Tape showed the worst performance for protecting solder joints ( $8 \%$ failures).

\section{Stiffness and Material Properties}

In general, the cotton fabric is less stiff than the polyester fabric. However, cotton fabric shrinks more after washing and drying than polyester fabric which could put more stress on the traces. Of all the protective materials, the BEMIS ${ }^{\mathrm{TM}}$ Seam Tape samples were the most flexible. The BEMIS ${ }^{\mathrm{TM}}$ Seam Tape was also water-resistant and translucent. Melco $^{\text {TM }}$ Seam Tape added little more stiffness than BEMIS $^{\text {TM }}$ Seam Tape, but was more flexible than both Heat n Bond ${ }^{\circledR}$ Iron-on Adhesive and Sil-Net ${ }^{\mathrm{TM}}$ Silicone Seam Sealer. Finally, Bondex ${ }^{\circledR}$ Fabric Mending Tape was the stiffest material used.

While there were benefits to the BEMIS ${ }^{\text {TM }}$ Seam Tape, some delamination was observed during wash and dry cycles, occasionally exposing LEDs and solder joints (Figure 10). This delamination clearly affected the protective properties that the material provided to traces and components.

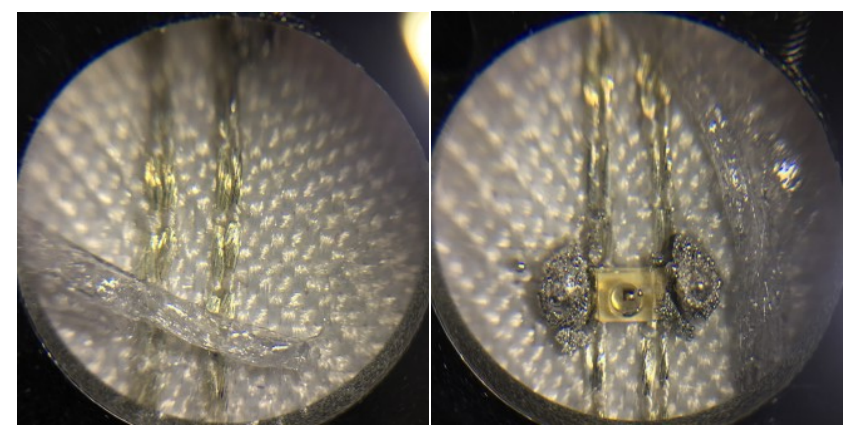

Figure 10. E1 delaminating after wash/dry cycles.

BEMIS $^{\text {TM }}$ Seam Tape has a thermoplastic adhesive layer on both sides, therefore when heated in the dryer it can fuse to itself or to other samples (Figure 11). This material fusing occurred during the beginning cycles and was not observed after the second cycle. It should be noted that specialist materials exist with thermoplastic adhesive on only one side of the tape: these were unfortunately not available to us, but would likely improve the performance of this material. Similarly, the sheer synthetic fabric used with the Heat $n$ Bond showed durability effects in terms of delamination over the cycle period.

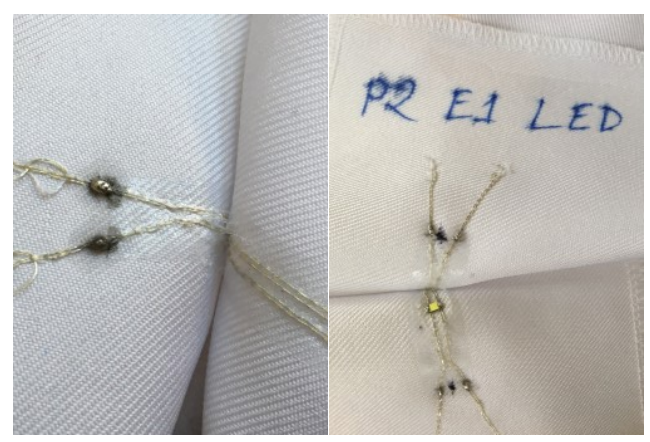

Figure 11. E1 bonding after wash/dry cycles.

\section{CONCLUSION}

This study evaluated the durability of stitched traces and surface-mount components during home laundering, under 
two different textile substrate conditions, and six different protective material conditions. Results showed that protective materials can effectively protect stitched circuits by maintaining trace resistance (best-case increase in resistance from 0.24 to $0.58 \Omega / \mathrm{m}$ ) and preventing breakage of solder joints (best-case $0 \%$ failure rate). Howeverifferences were seen in the relative performance of protective materials on trace resistance vs. solder joint durability. The material that was the most effective in protecting solder joints (silicone sealant) was also effective in maintaining polyester-LED trace conductivity. Conflicting results were seen for combinations of variables within the tape-type materials: Melco ${ }^{\text {TM }}$ Seam Tape provided best results for protecting traces with no electronics whereas BEMIS tape provided best results for protecting cotton-LED traces. Similarly, the material that was the least effective in protecting solder joints was the most effective in most cases while protecting stitched traces (seam tape film). Fusible tape-based products are more feasible to implement in cutand-sew factory environments, and several fusible tapes measured here added negligible stiffness to the stitched circuit.

However, overall the best-case approaches to protecting traces and circuits in each textile condition showed strong washability results. For polyester samples, $0 \%$ joint failure was observed for samples protected with Silicone Seam Sealer, and $143 \%(0.24 \Omega / \mathrm{m}$ to $0.58 \Omega / \mathrm{m})$ maximum change in resistance was observed for Melco ${ }^{\mathrm{TM}}$ Iron-on Seam Tape. For cotton samples $0 \%$ joint failure was observed for Silicone Seam Sealer and $171.78 \%(0.22 \Omega / \mathrm{m}$ to $0.60 \Omega / \mathrm{m})$ resistance increase for Melco ${ }^{\mathrm{TM}}$ Iron-on Seam Tape. These results showed an expectedly higher LED joint failure rate for un-protected samples (8\%) during water-based laundering compared to our previous study $(6 \%)$ evaluating un-protected samples in dry tumble testing [10]. However, here our results show that the use of the right protection material could improve durability for wet-laundered circuits above that of dry-tumbled unprotected circuits.

These results support the feasibility of tape-based approaches for protecting stitched circuit elements in textiles during washing. In future work, solutions that blend these capabilities (protecting joints from mechanical stresses, protecting traces from chemical interactions, and preserving hand-feel and manufacturability of the e-textile circuit) by leveraging different material properties of the encapsulation materials explored here should be investigated.

\section{ACKNOWLEDGMENTS}

This work was supported by the National Science Foundation under grant \#1253581.

\section{REFERENCES}

1. Mary E. Berglund, James Coughlin, Guido Gioberto, and Lucy E. Dunne. 2014. Washability of e-textile stretch sensors and sensor insulation. In Proceedings of the 2014 ACM International Symposium on Wearable Computers - ISWC '14, 127-128.

2. Mary E. Berglund, Julia Duvall, Cory Simon, and Lucy E. Dunne. 2015. Surface-mount component attachment for e- textiles. In Proceedings of the 2015 ACM International Symposium on Wearable Computers - ISWC '15, 65-66

3. Kunigunde Cherenack and Liesbeth van Pieterson. 2012. Smart textiles: Challenges and opportunities. Journal of Applied Physics 112, 9: 091301.

4. Nadine Dabby, Steven Girouard, Aleksandar Aleksov, Eric Lewallen, Sasha Oster, Racquel Fygenson, Braxton Lathrop, Michael Bynum, Mezhgan Samady, and Steven Klein. 2017. A scalable process for manufacturing integrated, washable smart garments applied to heart rate monitoring. In Proceedings of the 2017 ACM International Symposium on Wearable Computers ISWC '17, 38-41.

5. Francine Gemperle, Chris Kasabach, John Stivoric, Malcolm Bauer, and Richard Martin. 1998. Design for wearability. In Digest of Papers. Second International Symposium on Wearable Computers (Cat. No.98EX215), 116-122.

6. Qiao Li, and Xiao M. Tao. 2014. Three-dimensionally deformable, highly stretchable, permeable, durable and washable fabric circuit boards. Proceedings of the Royal Society A: Mathematical, Physical and Engineering Sciences 470, 2171: 20140472-20140472.

7. Linz Torsten, Christine Kallmayer, Rolf Aschenbrenner, and Herbert Reichl. 2005. Embroidering Electrical Interconnects with Conductive Yarn for The Integration of Flexible Electronic Modules into Fabric. In Ninth IEEE International Symposium on Wearable Computers (ISWC'05), 86-91.

8. I. Locher and A. G. Sefar. 2013. Joining technologies for smart textiles. In Multidisciplinary Know-How for Smart-Textiles Developers, Tünde Kirstein (ed.). Woodhead Publishing, 285305.

9. Corinne Mattmann, Tünde Kirstein, and Gerhard Tröster. 2005. A method to measure elongations of clothing. In Proc. 1st International Scientific Conference Ambience.

10. Md. Tahmidul Islam Molla, Steven Goodman, Nicholas Schleif, Mary E. Berglund, Cade Zacharias, Crystal Compton, and Lucy E. Dunne. 2017. Surface-mount manufacturing for e-textile circuits. In Proceedings of the 2017 ACM International Symposium on Wearable Computers - ISWC '17, 18-25.

11. Hojatollah R. Nejad, Meera P. Punjiya, and Sameer Sonkusale. 2017. Washable thread based strain sensor for smart textile. In 2017 19th International Conference on Solid-State Sensors, Actuators and Microsystems (TRANSDUCERS), 1183-1186.

12. Anne Schwarz, Ilda Kazani, L. Cuny, Carla Hertleer, F. Ghekiere, Geert De Clercq, Gilbert De Mey, and Lieva Van Langenhove.2011. Electro-conductive and elastic hybrid yarns The effects of stretching, cyclic straining and washing on their electro-conductive properties. Materials \& Design 32, 8: 4247 4256.

13. Sefar. Retrieved from: https://www.sefar.us/

14. Stretchable-circuits. Retrieved from: http://www.stretchablecircuits.com/

15. Xuyuan Tao, Vladan Koncar, Tzu-Hao Huang, Chien-Lung Shen, Ya-Chi Ko, and Gwo-Tsuen Jou.2017. How to Make Reliable, Washable, and Wearable Textronic Devices. Sensors, 673.

16. Lijing Wang. 2016. Performance Testing of Textiles: Methods, Technology and Applications. Woodhead Publishing.

17. Clint Zeagler, Scott Gilliland, Stephen Audy, and Thad Starner. 2013. Can I wash it? the effect of washing conductive materials usedin making textile based wearable electronic interfaces. In Proceedings of the 17th annual international symposium on International symposium on wearable computers - ISWC '13, 143. 